\title{
Preparation of a Novel Environmental Soybean Seed Coating Agent
}

\author{
Wenjin Wang, Defang Zeng \\ School of Resource and Environmental Engineering, Wuhan University of Technology, Wuhan, China \\ Email: 460978532@qq.com
}

How to cite this paper: Wang, W.J. and Zeng, D.F. (2018) Preparation of a Novel Environmental Soybean Seed Coating Agent. Journal of Agricultural Chemistry and Environment, 7, 133-142. https://doi.org/10.4236/jacen.2018.73012

Received: July 2, 2018

Accepted: July 29, 2018

Published: August 1, 2018

Copyright (c) 2018 by authors and Scientific Research Publishing Inc. This work is licensed under the Creative Commons Attribution International License (CC BY 4.0).

http://creativecommons.org/licenses/by/4.0/

(c) (i) Open Access

\begin{abstract}
In the study, a novel environmental soybean seed coating agent was made of natural polymer, trace elements, trace fertilizer and biopesticides etc., with the condition of high performance-to-price ratio and environmental friendliness. Effects of this soybean seed coating agent on soybean yield were tested through the laboratory and farm experiments. The tested results showed that the soybean yield using this seed coating agent was increased by $15.46 \%$, and its cost was decreased by $27.8 \%$, compared with the conventional soybean seed coating agent. It's nontoxic and harmless to human and animals, more friendly to the environment. So it has obvious economic and environmental benefits.
\end{abstract}

\section{Keywords}

Environmental Friendliness, Soybean Seed Coating Agent, Germination Tendency, Germination Percentage

\section{Introduction}

Soybean is famous for its abundant plant protein, which is one of the main economic crops in China. In order to ensure soybean could effectively against plant diseases and insect pests violations, in the northeast of China soybean seeds were coated with seed coating agent before it is planted [1]. But at present the mostly used soybean seed coating agent have serious toxicity and pollution to human body and the environment. For example, the Kangzhongcha and G Budweiser soybean coating agent, which is widely used at the Northeast market of China, is seriously toxicity and have pollution. The G Budweiser in the weight of $7 \mathrm{mg}$ can kill people and it have a lasting time for at least 50 years that cannot eliminate pollution in the environment [2]. The traditional toxicity soybean seed coating agent has become one of the major problems of ecological agriculture in China. 
Therefore, finding out a kind of safe and efficient novel environment-friendly soybean seed coating agent has become the problems that should be solved urgently in the field of agriculture and environmental protection. After many years of research [3] [4] [5], we finally developed a natural polymer material as the main ingredient, complemented with fertilizer, trace elements and biological pesticide and other additives to make a new efficient environmental friendly soybean seed. Comparing with traditional poisonous soybean seed coating agent, it can reduce the cost by $27.8 \%$ and increased the yield of soybean by $15.46 \%$. Meanwhile, it has no toxicity to human and animal, not pollute the environment and can increase grain output; it has significant economic and environmental benefit.

\section{Experimental Part}

\subsection{Main Experimental Instrument and Materials}

\subsubsection{Main Experimental Instrument}

Constant temperature and humidity incubator (WS-O1, Hubei Huangshi Hengfeng Medical Devices Co., Ltd.), high pressure steam sterilizer (YXQ-SG46-48SA Shanghai Boxun Industrial Co. Ltd.), temperature hygrometer (STH950, SUMMIT, USA), constant speed stirrer electronic (GS28B, Shanghai Anting electronic instrument factory), electronic balance (FA2004, Shanghai Yue Feng instrument Co., Ltd.), Petri dishes ( $\mathrm{d}=90 \mathrm{~mm}$, Shanghai Yuejin Medical Instrument Factory).

\subsubsection{Main Experimental Materials}

Natural organic polymer materials (PO, homemade), biological antibacterial agent (BT, Hubei Academy of Agricultural Sciences, biological pesticide production research center), glacial acetic acid, borax, sodium hydroxide, industrial ethylene glycol, trace elements, Potassium dihydrogen phosphate (they were all analytically pure, Hubei University Chemical Factory), sand, Natural Pigment Violet (Guangdong Shantou Mingde Food Additive Co., Ltd.), soybean seeds (goal nine), Kangzhongcha soybean seed coating agent (Harbin Long Chi Agricultural Chemical Co., Ltd.).

\subsection{Experiment Principle and Process}

\subsubsection{The Design Principle of Seed Coating Agent}

We used the non-toxic and harmless natural polymer materials (PO) to human and environment as the main raw ingredient for the soybean seed coating agent to replace the traditional toxic Budweiser grams. One of its characteristics is that it has filming ability, so it can be used as seed coating material. When it is mixed with other pesticides, growth regulators and plant fertilizer, it can be made of seed coating agent. The second characteristics is that it is rich in the element of $\mathrm{C}$ and $\mathrm{N}$, which can be used as microbial decomposition of plant growth and nutrient. Meanwhile, PO can change the soil microbial flora, which can promote 
the growth of micro organisms and inhibit plant pathogenic bacteria [6].

After all, the design of seed coating agent follows four principles, that is protect environmental, cheap, good quality and high yield. This coating material's cost is lower than the general market soybean seed coating agent's raw material costs (about 11 yuan $/ \mathrm{kg}$ ). We designed a number of film, antibacterial, fertilization, plant regulator, warning function and no pollution under the premise of seed coating formulation. The novel environment-friendly seed coating agent and traditional one were conducted experiments of various performance parameters, then we adjusted and optimized the formula on the original experiment, after many repeated adjustment experiments and optimization experiment, we ultimately determine the best seed coating agent, whose cost and environmental friendly properties are superior to other own-made seed coating agents and the traditional seed coating agent.

\subsubsection{Preparation of Seed Coating Agent}

The natural organic polymer materials (PO), biological antibacterial agent (BT), sodium hydroxide, ethylene glycol, pigment and borax were all respectively made into a certain concentration, then according to a certain proportion and order turn them into a container and mix well, stirring at constant speed electronic stirrer to stir for several minutes, the home-made soybean seed coating agent was finally been made.

\subsubsection{Seed Coating agent Laboratory Experimental}

Soybean seed coating agent in laboratory experimental refers to using the same soybean to be treated with environment-friendly seed coating agent or the traditional seed coating agent, and then placed the constant temperature and humidity incubator at the same conditions to be cultured, observing in their germination potential and germination rate, and the results were compared and analyzed. The specific process is as follows:

Preparation of seed coating agent: the self-made soybean seed coating agent and traditional soybean seed coating agent were according to a certain proportion respectively mixed with soybean seeds at room temperature, then hanging about 30 min to be coated, dried standby.

Laboratory germination experiments: using sand culture method, according to the International Seed Testing Association (ISTA) international rules for seed testing method [7], each seed coating agent selected 100 seeds. Firstly, dishes and sand used in the experiment were sterilized by high pressure steam sterilizer, then pad in $90 \mathrm{~mm}$ diameter circular filter paper culture dish and wet by water, according to the spatial distribution to put into 20 coated soybean seeds in each Petri dish. Every formula set three parallel samples, then covered with moist sand and the petri dish lid, put petri dish into constant temperature and humidity incubator whose temperature in $(28 \pm 1)^{\circ} \mathrm{C}$, humidity in $85 \%$ to culture the seeds. Investigated germination potential and germination rate for every $24 \mathrm{~h}$. According to the provisions of national rules for seed testing, calculate seed germination potential on the 3th day and the final seed germination rate on the 
7th day. The formula for calculating the germination potential and germination rate is as follows:

$$
\begin{aligned}
G E=\frac{G m}{G n} \times 100 \% & \text { Formula 2-1 } \\
G p=\frac{G a}{G n} \times 100 \% & \text { Formula 2-2 }
\end{aligned}
$$

Through the above two formulas to calculate the soybean seed's germination potential and germination rate, then comparing with the traditional one and the blank contrast on the two the indicators. If these two indicators are both higher than the traditional agent and reaches the maximum value then it is selected as a good formula in laboratory experiment. On this basis, by adjusting the value of $\mathrm{pH}, \mathrm{PO}$ molecular weight and concentration to further explore the optimal formula of seed coating agent in the laboratory and then we can carry out the field experiment.

\subsubsection{Seed Coating Agent Field Experimental}

Using the optimized and adjusted seed coating agent and the traditional seed coating agent to do field experiment in Northeast China, Jilin and Heilongjiang provinces. The soybean seed coating agent were mixed with the seeds according to a certain proportion, then swing and hanging about $30 \mathrm{~min}$ before sowing in the fields. Observed and calculated germination rate and yield of soybean and other information acquisition.

\section{Results and Discussion}

\subsection{Seed Coating Agent Laboratory Test Results}

Seed coating agent in the laboratory test results of the preliminary period is shown in Table 1. 1\# - 9\# is the homemade soybean seed coating agent, CK is the blank contrast group, traditional seed coating agent is Kangzhongcha soybean seed coating agent, which is currently recognized as the best soybean seed coating agent.

As can be seen from Table 1 that the main economic and technical indexes of the self-made seed coating agent $1 \#, 2 \#, 5 \#$ and $9 \#$ were better than that of traditional seed coating agent and CK, of which $5 \#$ had the best effect. Its germination potential and germination rate were better than the traditional seed coating agent, which were respectively increased by $12 \%, 5 \%$. Comparing with the CK, the germination potential and germination rate were respectively increased by $15 \%$ and $7 \%$. While the cost of self-made seed coating agent $5 \#$ decreased by $27.8 \%$ than traditional seed coating agent. Therefore, $5 \#$ was screened out as the best coating coating agent formula on the preliminary laboratory experiment.

\subsection{Seed Coating Agent Formula Laboratory Adjusted Results}

By changing the influence factors of $\mathrm{pH}$ value, $\mathrm{PO}$ molecular weight and concentration are based on the $5 \#$ formula to find out several seed coating agent whose performance is superior to the traditional seed coating agent. 
Table 1. The preliminary experimental results in laboratory.

\begin{tabular}{|c|c|c|c|c|c|c|c|c|c|c|c|}
\hline \multirow{2}{*}{$\begin{array}{c}\text { Comparative } \\
\text { index }\end{array}$} & \multicolumn{11}{|c|}{ Seed coating agent number } \\
\hline & $1 \#$ & $2 \#$ & $3 \#$ & $4 \#$ & $5 \#$ & $6 \#$ & $7 \#$ & $8 \#$ & 9\# & $\mathrm{CK}$ & Traditional \\
\hline $\begin{array}{l}\text { germination } \\
\text { potential/\% }\end{array}$ & $90 \pm 0.784$ & $88 \pm 0.856$ & $88 \pm 0.823$ & $67 \pm 1.031$ & $97 \pm 1.214$ & $80 \pm 1.237$ & $87 \pm 0.968$ & $70 \pm 0.811$ & $92 \pm 0.863$ & $82 \pm 0.902$ & $85 \pm 0.875$ \\
\hline $\begin{array}{l}\text { germination } \\
\text { rate/\% }\end{array}$ & $97 \pm 0.877$ & $97 \pm 0.874$ & $93 \pm 0.767$ & $80 \pm 0.826$ & $99 \pm 0.791$ & $92 \pm 0.874$ & $93 \pm 0.836$ & $95 \pm 0.951$ & $100 \pm 0.974$ & $93 \pm 1.023$ & $95 \pm 0.815$ \\
\hline
\end{tabular}

\subsubsection{The Effect of $\mathrm{pH}$ Value on the Main Performance Index of Seed Coating Agent}

Effect of $\mathrm{pH}$ value on the main performance index of seed coating agent are shown in Table 2. As can be seen from Table 2 that seed coating agent's best performance is at $\mathrm{pH}=6$. When $\mathrm{pH}$ were set from 4 to 6 , the seed coating agent's germination potential and germination rate were gradually increased with the increase of $\mathrm{pH}$ value. When $\mathrm{pH}=6$ the germination potential and germination rate reached the maximum value, then with the increase of $\mathrm{pH}$ value, the germination potential and germination rate were declined. Therefore the best $\mathrm{pH}$ value of the seed coating agent was 6 .

The reason why seed germination potential and germination rate were increased with $\mathrm{pH}$ value increased first and then decreased is that:

When the $\mathrm{pH}$ value is in low lever, the seed coating agent is acidic slightly stronger, the protective film formed on the surface of seeds was too closely, which influence the seed's permeability and seed in absorption of dissolved oxygen before germination, thus affecting the seed's seeding growth. With the increasing of $\mathrm{pH}$ value, the membrane binding were more suitable for seed's health growth. When the $\mathrm{pH}$ was at 6 , the protective film full played its breathable, waterproof effect, which ensured the seed can absorb oxygen and nutrients and provide favorable conditions for the healthy growth of the seed.

When the $\mathrm{pH}$ value is higher than 6 , it will affect the homemade coating to film, thus leading to the seed coating agent's functions of fertilizer, trace elements and antibacterial on seed promotion is blocked, and it is not healthy to the growth of the seed after sowing.

\subsubsection{Effect of Concentration on the Molecular Weight of PO to Main Performance Index of Seed Coating Agent}

Based on the 5\# formula $(\mathrm{pH}=6)$, keeping the other component and content remains unchanged, only change the natural polymer PO's molecular weight and concentration to research the main performance index of seed coating agent. Comparing the effect of molecular weight of PO in 150 thousand and 200 thousand on seed coating performance, the results are shown in Table 3.

From Table 3 it can be seen that the PO molecular weight in 200 thousand is better than 150 thousand. Therefore, the PO molecular weight in 200 thousand was chosen for the next experiment. In order to change the mass fraction of PO in the formula, to study the effects of different PO concentrations on the main performance index of seed. The result shown in Table 4. 
Table 2. Effect of $\mathrm{pH}$ value on the main performance index of seed coating agent.

\begin{tabular}{cccccccc}
\hline \multirow{2}{*}{ Comparative index } & \multicolumn{7}{c}{$\mathrm{pH}$ value } \\
\cline { 2 - 8 } & 4.0 & 4.5 & 5.0 & 5.5 & 6.0 & 6.5 & 7.0 \\
\hline \multirow{2}{*}{ germination potential/\% } & $85 \pm$ & $88 \pm$ & $92 \pm$ & $95 \pm$ & $98 \pm$ & $95 \pm$ & $95 \pm$ \\
& 0.556 & 0.618 & 0.453 & 0.703 & 0.577 & 0.859 & 0.710 \\
germination rate/\% & $90 \pm$ & $93 \pm$ & $97 \pm$ & $98 \pm$ & $99 \pm$ & $98 \pm$ & $97 \pm$ \\
& 0.943 & 1.026 & 0.837 & 0.939 & 0.908 & 0.790 & 0.516 \\
\hline
\end{tabular}

Table 3. Effect of PO molecular weight on the main performance index of seed coating agent.

\begin{tabular}{ccc}
\hline \multirow{2}{*}{ Comparative index } & \multicolumn{2}{c}{ PO molecular weight } \\
\cline { 2 - 3 } & 150 thousand & 200 thousand \\
\hline germination potential/\% & $92 \pm 1.026$ & $97 \pm 0.382$ \\
germination rate/\% & 971.015 & $99 \pm 0.943$ \\
\hline
\end{tabular}

Table 4. Effect of PO concentration on the main performance index of seed coating agent.

\begin{tabular}{ccccccc}
\hline \multirow{2}{*}{ Comparative index } & \multicolumn{5}{c}{ PO concentration } \\
\cline { 2 - 7 } & $0.1 \mathrm{~g} / \mathrm{L}$ & $0.2 \mathrm{~g} / \mathrm{L}$ & $0.5 \mathrm{~g} / \mathrm{L}$ & $1 \mathrm{~g} / \mathrm{L}$ & $1.5 \mathrm{~g} / \mathrm{L}$ & $2 \mathrm{~g} / \mathrm{L}$ \\
\hline germination potential/\% & $85 \pm 0.661$ & $87 \pm 0.516$ & $92 \pm 0.586$ & $98 \pm 0.732$ & $94 \pm 0.375$ & $87 \pm 1.290$ \\
germination rate/\% & $95 \pm 0.837$ & $97 \pm 0.908$ & $98 \pm 0.753$ & $99 \pm 0.943$ & $97 \pm 0.577$ & $95 \pm 0.453$ \\
\hline
\end{tabular}

As can be seen from Table 4, the PO concentration had obvious effects on the seed germination potential and germination rate. When the PO concentration were changed from the range $0.1 \mathrm{~g} / \mathrm{L}$ to $1 \mathrm{~g} / \mathrm{L}$, the seed germination potential and germination rate were increased with the concentration increased, when the concentration of $\mathrm{PO}$ is $1 \mathrm{~g} / \mathrm{L}$, we got the best germination condition. While the $\mathrm{PO}$ concentration is higher than $1 \mathrm{~g} / \mathrm{L}$, the seed germination potential and germination rate began to decline. Therefore, homemade seed coating agent's the best PO concentration was $1 \mathrm{~g} / \mathrm{L}$. This is because when the PO solution concentration is too high, it will cause a large coating viscosity, thus resulting the film is not uniform. When the PO concentration is too low, it will affect its film forming properties, which leads to membrane pore structure made of loose, and it is not conducive to the healthy growth of the seed.

\subsection{Optimization and Comparison Results}

Results optimized homemade seed coating agent's formula 5-F\# and blank control (CK) and traditional seed coating agent are shown in Table 5.

As can be seen from Table 5 that germination potential and germination rate were respectively increased by $16 \%$ and $8 \%$ comparing with the blank contrast group. Increased by $13 \%$ and $5 \%$ comparing with the traditional seed coating agent, and the cost were decreased by $27.8 \%$ comparing with the traditional seed coating agent, which significantly have the price advantage. The seed coating 
Table 5. Experimental results of optimization and comparison.

\begin{tabular}{cccc}
\hline \multirow{2}{*}{ Comparative index } & \multicolumn{3}{c}{ samples } \\
\cline { 2 - 4 } & $5-\mathrm{F} \#$ & $\mathrm{CK}$ & traditional one \\
\hline The drug cost/yuan $\mathrm{kg}^{-1}$ & $7.9 \pm 0.710$ & 0 & $11.0 \pm 1.214$ \\
germination potential/\% & $98 \pm 0.874$ & $82 \pm 0.407$ & $85 \pm 0.796$ \\
germination rate/\% & $99 \pm 0.811$ & $92 \pm 1.204$ & $95 \pm 1.237$ \\
\hline
\end{tabular}

treatment on soybean seed is shown in Figure 1. Their germination potential is shown in Figure 2.

The seed root length of the best formula is about the same with the original formula, their stability degree is higher than the blank sample. But the best formula's coated seeds is stronger than the original formula, fibrous root length reached 2 times than the original recipe (Figure 3), while the blank sample of fibrous root is very short. In addition, disease appears in soybean blank samples (Figure 4), this situation has never occurred in the coated samples.

As can be seen from Figure 3 that being coated with seed coating agent, those seeds' germination potential is obviously superior to the traditional seed coating agent.

\subsection{The Field Test Results}

The soybean seed were respectively coated with 5-F\# seed coating agent and the traditional seed coating agent, and then sowed in three different experimental bases on the northeast of Jilin and Heilongiang two provinces. We also set a blank contrast group, and all the seed were under the same conditions of planting. The results are shown in Table 6.

As can be seen from Table 6 that seed germination rate of the self-made seed coating agent formula 5 -F\# was increased by $22 \%$ than the blank contrast group. Comparing with the traditional seed coating agent, the seed germination rate of 5 -F\# was increased by $13 \%$, and the price declined by $20 \%$, so every acres of seed coating agent cost is reduced by $24.2 \%$. Meanwhile the yield of soybean was increased by $15.46 \%$ compared with traditional coating agent, and it is safe, non-toxic and no pollution. What's more, the results of laboratory seed germination potential, germination rate and field experiments were have the same trend. Therefore, the 5-F\# formulation of seed coating agent is environmental protection, high quality and high yield and those three characteristics were confirmed in the field experiment, so it has important application value.

\subsection{Mechanism Discussion on the Environment Seed Coating Agent}

Being treated with seed coating agent, PO can promote the synthesis of m-RNA in seeds, it can greatly enhance the enzyme activity in the seeds' germination and improve the seed germination rate, seedling growth. Used PO on the concentration of $1 \%$ to coated with the soybean seeds can promote the formation of pea 


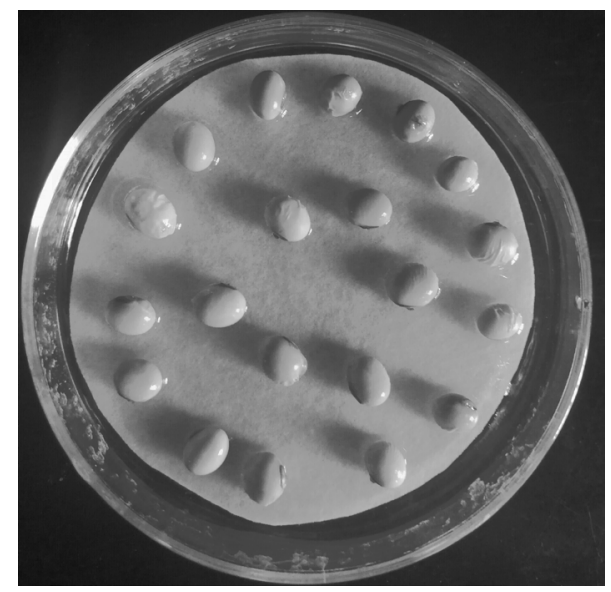

Figure 1. Seed coating treatment on soybean seed.

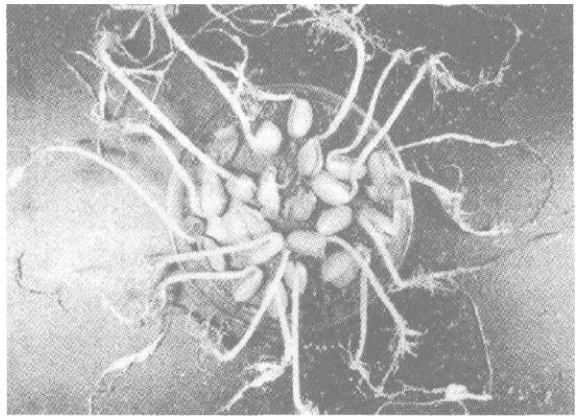

(a)

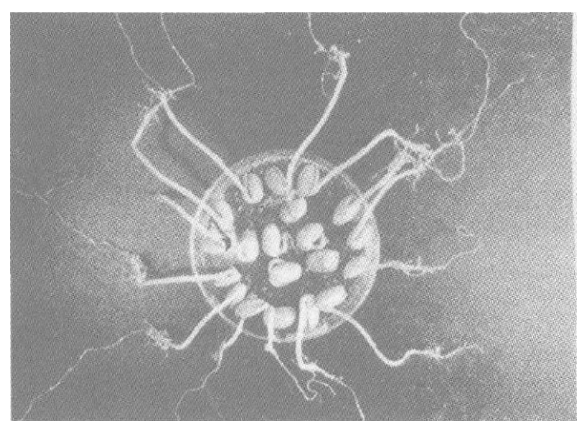

(b)

Figure 2. Seed germination comparison of the homemade and traditional seed coating agent. (a) Germination potential of homemade seed coating agent; (b) Germination potential of traditional seed coating agent.



Figure 3. The upper is the best homemade formula sample, the under one is the traditional seed coating agent samples.

peptide containing phenolic compounds, increase the synthesis rate of phenylalanine. In sixth days after seed germination, the enzyme activity can be increased by 1.2 to 1.7 times than that of the untreated one, which greatly promoted the growth of soybean. 




Figure 4. The diseased soybean blank samples.

Table 6. Comparison results of field experiment.

\begin{tabular}{cccc}
\hline \multirow{2}{*}{ Comparative index } & \multicolumn{3}{c}{ samples } \\
\cline { 2 - 4 } & 5 -F\# & CK & traditional one \\
\hline Germination rate/\% & 98 & 76 & 85 \\
Yeild per 667 square meters $\cdot \mathrm{kg}^{-1}$ & 265 & 208 & 224 \\
Agent usage mount/g.667 square meters ${ }^{-1}$ & 88 & - & 95 \\
Agent cost/yuan.667 square meters ${ }^{-1}$ & 2.5 & - & 3.3 \\
Agent price/yuan $\cdot \mathrm{kg}^{-1}$ & 28 & - & 35 \\
Toxic & non & non & serious \\
\hline
\end{tabular}

Seed coating agent made by the natural polymer PO had good film forming ability, the protective film for coated seeds can form a layer of dense, thus keeping the seeds of nutrients within the minimum costs. When the coated seeds implanted into the soil, the polymer membrane can swelling the water from soil, which showed it has good permeability, so that the seeds to fully absorb the dissolved oxygen in the soil, and the rapid release of $\mathrm{CO}_{2}$.

The natural polymer PO can make plants produce systemic antibody, induce the resistance factor, which in return improve the host defense response, increase the disease resistant ability, help the soybean keep effective prevention and treatment of fungal, bacterial and viral invasion, thus resulting in a certain repellent effect of plant diseases and insect pests in the soil. Due to the addition of the repellent BT, so the repellent effect will guarantee the healthy growth of each plant species.

The natural polymer PO could be used as novel inhibitors of plant diseases, it can induced disease resistance of plant, such as the leaf Tofu pudding, soybean root rot disease [8]. What's more, $\mathrm{PO}$ is an elicitor of plant resistance which can also regulate the changes of enzyme activity and disease resistance of plants, and have a direct inhibitory effect on many plant pathogenic fungi. In addition, the film also prevents direct contact with pathogens and host tissues or cells, thereby it will reducing the effective penetration of pathogenic bacteria. 


\section{Conclusion}

Using this natural organic polymer PO as main ingredient, complemented by trace elements and other additives, we prepared a novel environment-friendly soybean seed coating agent. In the process of preparation, keeping the coating $\mathrm{pH}$ value adjust to 6 , the molecular weight of natural polymer PO in 200 thousand and its concentration is controlled at about $1 \%$, can achieve the best effect of increasing yield. Comparing with the traditional soybean seed coating agent, its seed yield increased by $15.46 \%$; the cost is reduced by $27.8 \%$, and it is safety, non-toxic and pollution-free. It not only has significant economic and environmental benefits to farmers, but also can protect the environment. Therefore, the seed coating agent is a good product benefits to the country, in China's vast rural areas (especially in the Northeast producing soybean) has important application value.

\section{References}

[1] Pan, L.G., Tao, L.M., Liu, T.R., et al. (2005) Seed Coating and Its Key Technology. The Pesticide (Chinese Pesticide), 33, 437-442.

[2] Xu, W.L., Chen, Y.F. and Wu, G.Q. (1999) The Synthesis of Seed Coating Agent and Its Properties Research. SEED, 3, 11-12.

[3] Herbert, R.M. (2003) Application of Latex Emulsion Polymers in Seed Coating Technology. Pesticide Fomailations and Application Systems, 23, 62-63.

[4] Ren, X.R., Xu, H.T. and Xu, B. (2002) Development of Soybean Be Imperative. Henan Science and Technology, 3, 7.

[5] Wu, X.H., Liu, X.L., Wang, H.M., et al. (2003) Research Progress of Coating Agents in China. Pesticide, 42, 1-5.

[6] Zhu, J.J. (2002) Development of Soybean Wet Mixing Agent. PFST ICIDES, 41, 12-13.

[7] Kang, G.H., Feng, Y.R., Yu, C.X., et al. (2005) The Effects of Different Seed Coating Agents on Seed Vigor and Plant Growth of Soybean. Shandong Agricultural Sciences, 3,42 .

[8] Wang, G.W. and Han, X.D. (2010) Chitosan on Seed Germination of Several Cruciferous Vegetables. Seeds, 29, 99-101. 